\title{
Outbreak of late-onset Group B Streptococcal disease with serotype Ib in a Neonatal Intensive Care Unit
}

\author{
Hiroko Horie-Ota ${ }^{\oplus}$, Kazushige Ikeda ${ }^{\oplus}$ \\ Division of Neonatology, Department of Pediatrics, Saitama City Hospital, Saitama, Japan.
}

\begin{abstract}
Background. Hospital outbreaks of invasive group B streptococcus (GBS) infection are rare. There are only a few published reports of late-onset GBS outbreaks in neonatal intensive care units (NICUs). We report here three cases of late-onset GBS in our NICU.

Case. Three preterm very low birth weight (VLBW) infants born at $24-27$ weeks gestation developed lateonset GBS sepsis within four weeks. Two asymptomatic GBS carriers were identified in the NICU prior to the outbreak. Tests of maternal rectovaginal GBS colonization were negative in all three cases; as such, vertical transmission was unlikely. All three GBS isolates were capsular serotype 1b, with comparable antibiotic susceptibility profiles.
\end{abstract}

Conclusion. Preterm delivery and VLBW are associated with an increased risk of invasive late-onset GBS infection. This report underscores the ongoing risk of nosocomial transmission of GBS in the NICU.

Key words: Group B Streptococcus, sepsis, outbreak, very low birth weight infant, NICU.

Group B streptococcus (GBS) is a leading cause of neonatal sepsis and meningitis and accounts for $50-60 \%$ of all cases in Japan. Infant invasive GBS disease is classified as early-onset disease if it occurs within the first 6 days of life; by contrast, late-onset disease (LOD) develops seven or more days after birth. ${ }^{1}$ The frequency of earlyonset GBS infection has decreased substantially since the introduction of routine screening for maternal rectovaginal GBS colonization and the use of intrapartum antibiotic prophylaxis (IAP). ${ }^{2}$ However, late-onset GBS infection is unaffected by chemoprophylaxis and continues to cause significant morbidity and mortality in newborns. The pathogenesis of late-onset GBS infection remains controversial ${ }^{3}$; there are only a few published studies reporting outbreaks of late-onset GBS specifically in neonatal intensive care units (NICUs). ${ }^{4}$ In this report, we describe

\footnotetext{
$凶$ Kazushige Ikeda

kazuiked@keio.jp
}

Received 23rd April 2020, revised 20th August 2020, accepted 5th November 2020. a cluster of nosocomial LOD GBS infections in a regional NICU that were diagnosed over a period of four weeks involving three very low birth weight (VLBW) infants.

Patient presentations have been given in Table I.

\section{Case 1}

A male infant weighing $722 \mathrm{~g}$ was delivered by cesarean section at $246 / 7$ weeks of gestation who required immediate resuscitation via endotracheal intubation at birth. Maternal rectovaginal GBS colonization was negative, although preterm premature rupture of the membranes (PPROM) had been recognized at $234 / 7$ weeks of gestation. The patient serum IgM was detected at $33 \mathrm{mg} / \mathrm{dL}$, suggesting intrauterine infection. Antibiotic therapy with ampicillin and gentamicin was initiated at birth (day 0 ) and continued through postnatal day 5; hydrocortisone was also administered (postnatal days 0 - 9) to prevent bronchopulmonary dysplasia. Mechanical ventilation was discontinued on postnatal day 40; however, on 
Table I. Characteristics of the three patients with LOD in the NICU.

\begin{tabular}{lccc}
\hline & Case 1 & Case 2 & Case 3 \\
\hline Sex & Male & Male & Male \\
Gestational age (weeks) & $246 / 7$ & $261 / 7$ & $275 / 7$ \\
Birth weight (grams) & 722 & 620 & 1158 \\
Delivery & Cesarean & Cesarean & Cesarean \\
Apgar score $(1 / 5 \mathrm{~min})$ & $3 / 7$ & $4 / 6$ & $6 / 5$ \\
Onset $($ days after birth) & 55 & 19 & 43 \\
WBC $(/ \mu \mathrm{L})$ & 4,820 & 10,700 & 10,250 \\
C-reactive protein $(\mathrm{mg} / \mathrm{dL})$ & 1.66 & 0.17 & 2.55 \\
Blood culture & GBS + & GBS + & GBS + \\
CSF culture & Negative & Negative & $($ Not done) \\
Maternal rectovaginal culture & GBS - & GBS - & GBS - \\
Maternal milk culture & GBS + & GBS - & GBS - \\
\hline
\end{tabular}

WBC: white blood cell, CSF: cerebrospinal fluid, GBS: group B Streptococcus.

postnatal day 55, he again required respirator support due to frequent apnea. GBS was detected in cultures of peripheral blood on the same day; cerebrospinal fluid cultures remained negative. Of note, weekly surveillance cultures of both stool and respiratory secretions were negative for GBS before the infection. GBS was later found in maternal breast milk.

\section{Case 2}

A male infant weighing $620 \mathrm{~g}$ was delivered by emergency cesarean section at $261 / 7$ weeks of gestation because of suspected placental abruption. Maternal rectovaginal GBS colonization was negative. He was intubated immediately after birth and provided with surfactant replacement therapy; hydrocortisone was administered for hypotension. He was extubated on postnatal day 4. On postnatal day 19, he developed lethargy and poor feeding behavior; his laboratory tests were notable for hypoglycemia and an elevated level serum C-reactive protein. He was diagnosed with late-onset GBS sepsis; GBS was detected in blood but not in cerebrospinal fluid cultures. Weekly surveillance cultures from stool were GBS positive for the first time on day 5 of the infection. GBS was not detected in maternal breastmilk.

\section{Case 3}

A male infant weighing 1,158 $\mathrm{g}$ was delivered by cesarean section at $275 / 7$ weeks of gestation due to premature uterine contractions associated with fetal footling presentation. Maternal rectovaginal GBS colonization was negative. $\mathrm{He}$ was admitted to the NICU where he underwent mechanical ventilation from $0-2$ days of age. Frequent apnea was noted on postnatal day 43 associated with an elevated serum level of C-reactive protein. GBS was detected in his blood on the same day; his overall condition precluded sampling and evaluation of his cerebrospinal fluid. His weekly surveillance cultures were negative for GBS before the diagnosis of bacterial sepsis. GBS was not found in maternal breastmilk.

Informed consent was obtained from the parents of all three patients featured in this study.

\section{Discussion}

We report here three cases of preterm VLBW infants who developed LOD GBS sepsis in the NICU within four weeks (Fig. 1). Serotype analysis of the strains isolated from the infants and from maternal breast milk revealed that all samples were capsular type $\mathrm{Ib}$; interestingly, this capsule type is not typically associated with 


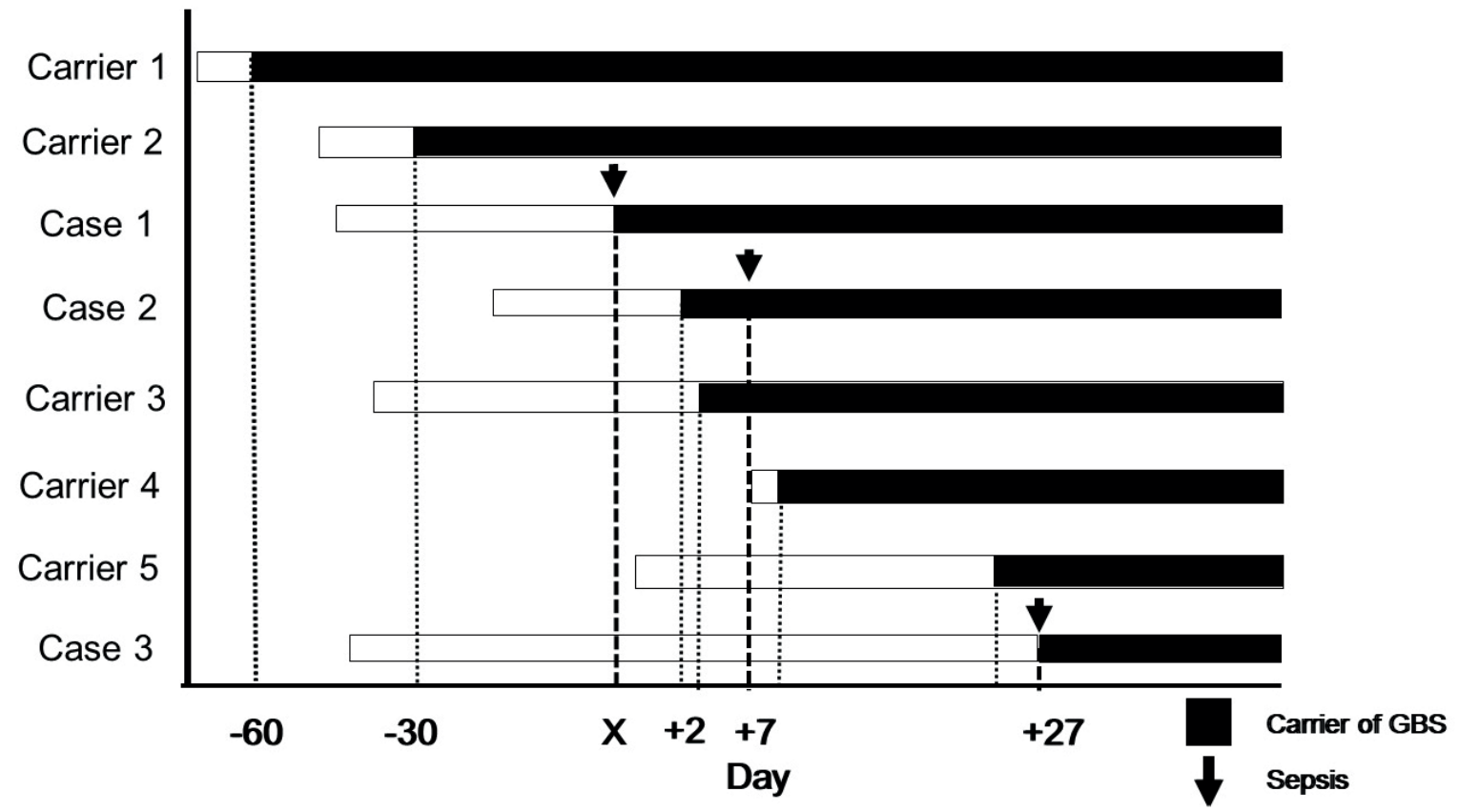

Fig. 1. Time course of the outbreak. Black arrows indicate the onset of LOD in each patient. Black bands represent the period of GBS carrier.

LOD (Table II) ${ }^{5}$ The antibiotic susceptibility profiles of all isolates were comparable to one another.

In this report, maternal rectovaginal GBS colonization was negative throughout; as such, a mechanism involving vertical transmission of this pathogen was unlikely. Bacterial colonization surveys of all the neonates in the NICU were performed routinely on a weekly basis. Of note, two asymptomatic infants were identified in the NICU prior to the development of GBS sepsis in the index case (Fig. 1; carriers 1 and 2). Patient case 2 was identified as a GBS carrier prior to the onset of sepsis; however, two of the three patients (patient cases 1 and 3) were not GBS carriers before developing LOD. Of note, an additional three new carriers (carriers 3,4 , and 5) were identified in the NICU after the onset of disease observed in patient case 1. After the outbreak, we conducted similar prevalence surveys focused on possible sources of GBS infection, including equipment (i.e., milk warmers, feeding bottles, and shared breast pumps). No GBS was detected in any swab samples from NICU equipment. Breast milk from the mother of patient case 1 was later identified as positive for GBS. On the other hand, maternal breastmilk from patients 2 and 3 was negative. Given these findings, we hypothesize that patient case 1 may have acquired the infection from maternal milk or asymptomatic carriers; cases 2 and 3 may have developed GBS sepsis via transmission from an asymptomatic carrier on the medical staff.

NICU-associated outbreaks of invasive GBS disease are rare and the mechanisms associated with transmission and development of severe LOD remain controversial. One group has reported that more than half the mothers of infants who develop LOD are carriers of GBS. ${ }^{3}$ Routine screening for maternal GBS carriage at 35 to 37 weeks' gestation and consequent IAP may not eradicate maternal colonization; as such, the mother may remain a source of GBS. However, horizontal transmission from nosocomial sources has also been reported. ${ }^{6}$

At this time, there are only a few published reports of GBS outbreaks in NICUs. Boyer reported that $34 \%$ of the nursing staff were identified as GBS carriers in association with 
Table II. The susceptibility of antibiotics and capsular serotype of isolated GBS in each patient.

\begin{tabular}{|c|c|c|c|c|c|c|}
\hline & Penicillin & Ampicilin & Erythromycin & Clindamycin & Levofloxacin & Capsular serotype \\
\hline $\begin{array}{l}\text { Case } 1 \\
\text { (blood) }\end{array}$ & $S$ & $S$ & $\mathrm{R}$ & $\mathrm{R}$ & $\mathrm{R}$ & - \\
\hline $\begin{array}{l}\text { Case } 1 \\
\text { (pharyngeal mucosa) }\end{array}$ & S & $S$ & $\mathrm{R}$ & $\mathrm{R}$ & $\mathrm{R}$ & $\mathrm{Ib}$ \\
\hline $\begin{array}{l}\text { Case } 1 \\
\text { (maternal milk) }\end{array}$ & $S$ & S & $\mathrm{R}$ & $\mathrm{R}$ & $\mathrm{R}$ & $\mathrm{Ib}$ \\
\hline $\begin{array}{l}\text { Case } 2 \\
\text { (blood) }\end{array}$ & $S$ & $S$ & $\mathrm{R}$ & $\mathrm{R}$ & $\mathrm{R}$ & - \\
\hline $\begin{array}{l}\text { Case } 2 \\
\text { (stool) }\end{array}$ & $S$ & $S$ & $\mathrm{R}$ & $\mathrm{R}$ & $\mathrm{R}$ & $\mathrm{Ib}$ \\
\hline $\begin{array}{l}\text { Case } 3 \\
\text { (blood) }\end{array}$ & $S$ & $S$ & $\mathrm{R}$ & $\mathrm{R}$ & $\mathrm{R}$ & $\mathrm{Ib}$ \\
\hline $\begin{array}{l}\text { Carrier } 1 \\
\text { (stool) }\end{array}$ & $S$ & $S$ & $\mathrm{R}$ & $\mathrm{R}$ & $\mathrm{R}$ & $\mathrm{Ib}$ \\
\hline $\begin{array}{l}\text { Carrier } 2 \\
\text { (stool) }\end{array}$ & S & S & $\mathrm{R}$ & $\mathrm{R}$ & $\mathrm{R}$ & $\mathrm{Ib}$ \\
\hline $\begin{array}{l}\text { Carrier } 3 \\
\text { (stool) }\end{array}$ & $S$ & S & - & - & $\mathrm{R}$ & $\mathrm{Ib}$ \\
\hline $\begin{array}{l}\text { Carrier } 4 \\
\text { (stool) }\end{array}$ & S & S & $\mathrm{R}$ & $\mathrm{R}$ & $\mathrm{R}$ & - \\
\hline $\begin{array}{l}\text { Carrier } 4 \\
\text { (pharyngeal mucosa) }\end{array}$ & S & S & $\mathrm{R}$ & $\mathrm{R}$ & - & $\mathrm{Ib}$ \\
\hline $\begin{array}{l}\text { Carrier } 5 \\
\text { (stool) }\end{array}$ & S & S & $\mathrm{R}$ & $\mathrm{R}$ & $\mathrm{R}$ & $\mathrm{Ib}$ \\
\hline
\end{tabular}

S: susceptible, R: resistant.

a cluster outbreak of this infection. ${ }^{7}$ Recently, Collin reviewed the overall incidence and prevalence of GBS clusters in healthcare settings, and identified only 12 reports focused on neonatal LOD over 50-year period. ${ }^{4}$

Infant-to-infant or staff member-to-infant spread may occur via physical contamination. Cross-transmission has been associated with high staff workloads, patient crowding and deficient cot-spacing and/or high patient-tonurse ratios. ${ }^{4}$ Likewise, poor infection control practices, including inadequate hand hygiene and care taken when preparing infant formula feedings are magnified in premature infants who are then exposed to GBS. Environmental contamination from inadequate disinfection of equipment or surfaces is also appreciated as a reservoir for invasive pathogens. Shared breast pumps, laryngoscope blades, patient monitors, and other surfaces that are handled frequently have all been implicated as sources of infection. ${ }^{8}$

While breast milk plays an important role in protecting infants against GBS infection, it may also represent a significant source of pathogens associated with LOD and infection. ${ }^{9}$ It has been estimated that $\sim 0.8$ to $3.5 \%$ of mothers carry GBS in their breast milk; this pathogen is among the more common causes of mastitis. However, the role of breast milk and its role in promoting neonatal LOD remains incompletely understood..$^{10}$ In this report, breast milk from the mother of the index case (patient case 1) was GBS positive. As such, breast milk was suspected to be one of the potential sources of GBS LOD in this patient. 
Several published reports have defined risk factors associated with LOD.,11 We note that all three cases featured here were VLBW infants born prematurely, from 24 to 27 weeks of gestation, and developed GBS sepsis at postnatal days 19-55 days after birth (Table I). Preterm delivery and low birth weight have been associated with an increased risk of invasive GBS infection and LOD. Important factors that influence progression from GBS colonization to infection include immune status and integrity of the gastrointestinal mucosa as well as the bacterial load and virulence. Compared with infants born at term, preterm infants typically have a more poorly-developed immune system as well as transient hypogammaglobulinemia. The other sources of infection associated with LOD include the use of invasive devices as well as administration of steroids ${ }^{12}$ that may be required for life-supporting care.

Other than scrupulous attention to hygiene and infection control practices, there are no known mechanisms that might be put in place to prevent the spread of GBS. Infection control practices typically include standard precautions such as hand hygiene, careful preparation and thawing of frozen breast milk, and weekly surveillance of bacterial colonization; all of these measures are critical toward efforts to prevent GBS LOD and sepsis in the NICU. Physicians and nursing staff need to be alert to the presence of GBS in routine surveillance cultures, as well as other pathogens, including multiple drug-resistant bacteria, such as methicillinresistant Staphylococcus aureus. To prevent GBS outbreaks in the NICU, Jauneikaite and colleagues note that a single case of GBS LOD should be considered as a sentinel of a future outbreak $^{13}$; all cases of GBS infection should prompt enhanced prospective and retrospective surveillance throughout the facility.

There are two limitations to our findings. First, although we provide some information about potential routes of GBS transmission, the precise mode of transmission was not fully determined because we did not perform surveillance on the healthcare personnel who were in the NICU during this period. Second, while our results may be suggestive of a single source, this could not be concluded with certainty without molecular typing information for each bacterial isolate. ${ }^{14}$

In summary, we report here an outbreak of GBS LOD among VLBW preterm infants in a NICU. In a recent publication, Collins reported that the risks associated with GBS outbreaks specifically in the setting of the NICU have increased over the past 9 years. ${ }^{4}$ Indeed, some reports recommended that a single case of GBS LOD might be treated as a sentinel, and should be followed by enhanced surveillance measures. ${ }^{13}$ This report underscores the need to be aware of the risk of nosocomial transmission of GBS infection in the NICU and the need to be alert to any indication of GBS contamination in routine surveillance cultures.

\section{Acknowledgment}

The authors thank Dr. Miyuki Morozumi, Department of Infectious Diseases, Keio University School of Medicine, Tokyo, for capsular serotype analyses and Dr. Hiroyuki Shiro, Department of Pediatrics, Yokohama Rosai Hospital, Yokohama, for precious advice on the infectious control in NICU.

\section{Author contribution}

The authors confirm contribution to the paper as follows: clinical data and preparation of the manuscript: $\mathrm{HH}$; study conception and design: KI. All authors reviewed the results and approved the final version of the manuscript.

\section{Conflict of interest}

The authors declare no conflict of interest. 


\section{REFERENCES}

1. Nanduri SA, Petit S, Smelser C, et al. Epidemiology of invasive early-onset and late-onset Group B Streptococcal disease in the United States, 2006 to 2015: Multistate Laboratory and Population-Based Surveillance. JAMA Pediatr 2019; 173: 224-233.

2. Mukhopadhyay S, Puopolo KM. Preventing Neonatal Group B Streptococcus disease: the limits of success. JAMA Pediatr 2019; 173: 219-220.

3. Berardi A, Rossi C, Lugli L, et al. Group B streptococcus late-onset disease: 2003-2010. Pediatrics 2013; 131: e361-e368.

4. Collin SM, Lamb P, Jauneikaite E, et al. Hospital clusters of invasive Group B Streptococcal disease: a systematic review. J Infect 2019; 79: 521-527.

5. Madrid L, Seale AC, Kohli-Lynch M, et al; Infant GBS Disease Investigator Group. Infant Group B Streptococcal disease incidence and serotypes worldwide: systematic review and meta-analyses. Clin Infect Dis 2017; 65(Suppl 65): S160-S172.

6. Al-Maani A, Streitenberger L, Clarke M, et al. Nosocomial transmission of group B streptococci proven by positive environmental culture. Oman Med J 2014; 29: 376-379.

7. Boyer KM, Vogel LC, Gotoff SP, Gadzala CA, Stringer J, Maxted WR. Nosocomial transmission of bacteriophage type 7/11/12 group B streptococci in a special care nursery. Am J Dis Child 1980; 134: 964-966.
8. Barbadoro P, Marigliano A, Savini S, D'Errico MM, Prospero E. Group B Streptococcal sepsis: an old or ongoing threat? Am J Infect Control 2011; 39: e45-e48.

9. Olver WJ, Bond DW, Boswell TC, Watkin SL. Neonatal group B streptococcal disease associated with infected breast milk. Arch Dis Child Fetal Neonatal Ed 2000; 83: F48-F49.

10. Davanzo R, De Cunto A, Travan L, Bacolla G, Creti R, Demarini $S$. To feed or not to feed? Case presentation and best practice guidance for human milk feeding and group B streptococcus in developed countries. J Hum Lact 2013; 29: 452-457.

11. MacFarquhar JK, Jones TF, Woron AM, et al. Outbreak of late-onset group B Streptococcus in a neonatal intensive care unit. Am J Infect Control 2010; 38: 283-288.

12. Poets CF, Lorenz L. Prevention of bronchopulmonary dysplasia in extremely low gestational age neonates: current evidence. Arch Dis Child Fetal Neonatal Ed 2018 ;103: F285-F291.

13. Jauneikaite E, Kapatai G, Davies F, et al. Serial clustering of late-onset Group B Streptococcal infections in the Neonatal Unit: a genomic reevaluation of causality. Clin Infect Dis 2018; 67: 854860.

14. Berardi A, Guidotti I, Creti R, et al. Two overlapping clusters of Group B streptococcus late-onset disease in a Neonatal Intensive Care Unit. Pediatr Infect Dis J 2018; 37: 1160-1164. 